\title{
ON DIFFERENTIAL SUBORDINATION, SUPERORDINATION AND SANDWICH THEOREMS OF SYMMETRIC ANALYTIC FUNCTIONS
}

\author{
BY
}

\author{
ALI MUHAMMAD
}

\begin{abstract}
In this paper, we study the interesting properties of differential subordination and superordination for the classes of symmetric functions analytic in the unit disc. We derive sandwich results on the basis of this theory.

Mathematics Subject Classification 2010: 30C45, 30C50.

Key words: analytic functions, convex functions, symmetric functions, differential subordination and superordination.
\end{abstract}

\section{Introduction}

Let $H(E)$ denote the class of analytic functions in the open unit disc $E=\{z: z \in \mathbb{C}$ and $|z|<1\}$ and let $H[a, 1]$ denote the subclass of the functions $f \in H(E)$ of the form $f(z)=a+a_{1} z+a_{2} z^{2}+\ldots, a \in \mathbb{C}$. Also, let $\mathcal{A}$ be the subclass of functions $f \in H(E)$ of the form

$$
f(z)=z+\sum_{k=2}^{\infty} a_{k} z^{k} .
$$

If $f$ and $g$ are analytic in $E$, we say that $f$ is subordinate to $g$, written $f \prec g$ or $f(z) \prec g(z)$, if there exists a Schwarz function with $w(0)=0$ and $|w(z)|<1$ in $E$ such that $f(z)=g(w(z))$.

Suppose that $h$ and $k$ are two analytic functions in $E$ let $\varphi(r, s, t ; z)$ : $\mathbb{C}^{3} \times E \rightarrow \mathbb{C}$. If $h$ and $\varphi\left(h(z), z h^{\prime}(z), z^{2} h^{\prime \prime}(z) ; z\right)$ are univalent functions in $E$ and if $h$ satisfies the second order superordination

$$
k(z) \prec \varphi\left(h(z), z h^{\prime}(z), z^{2} h^{\prime \prime}(z) ; z\right),
$$


then $k$ is said to be a solution of the differential superordination (1.2). A function $q \in H(E)$ is called a subordinant to $(1.2)$, if $q(z) \prec h(z)$ for all the functions $h$ satisfying (1.2).

A univalent subordinant $\widetilde{q}$ that satisfies $q(z) \prec \widetilde{q}(z)$ for all of the subordinants $q$ of (1.2), is said to be the best subordinant.

Recently, Miller and Mocanu [7] obtained sufficient conditions on the functions $k, q$ and $\varphi$ for which the following implications hold $k(z) \prec$ $\varphi\left(h(z), z h^{\prime}(z), z^{2} h^{\prime \prime}(z) ; z\right) \Longrightarrow q(z) \prec h(z)$. Using these results, the authors in [1] considered certain classes of first-order differential superordinations, see also [4], as well as superordination-preserving integral operators [3]. AOUF ET AL. [1, 2], obtained sufficient conditions for certain normalized analytic functions $f$ to satisfy $q_{1}(z) \prec \frac{z f^{\prime}(z)}{f(z)} \prec q_{2}(z)$, where $q_{1}$ and $q_{2}$ are given univalent normalized functions in $E$. Very recently, Shanmugam ET AL. $[12,13]$ obtained the such called sandwich results for certain classes of analytic functions. For interested readers we refer to the work done by the authors $[1,8,9,11]$.

In [14], SAKAGUCHI defined the class of starlike functions with respect to symmetrical points as follows:

Let $f \in \mathcal{A}$. Then $f$ is said to be starlike with respect to symmetrical points in $E$ if, and only if, $\Re e \frac{z f^{\prime}(z)}{f(z)-f(-z)}>0, z \in E$. Obviously, it forms a subclass of close-to-convex functions and hence univalent. Moreover, this class includes the class of convex functions and odd starlike functions with respect to the origin, see [14].

In this paper, we study several applications of the theory of differential subordination and superordination for normalized univalent symmetric analytic functions in the unit disc. On the basis of this theory we also investigate some important sandwhich results of symmetric analytic functions which will be useful in obtaining applications of geometric function theory.

\section{Preliminary results}

Definition 2.1 ([7]). Let $Q$ be the set of all functions $f$ that are analytic and injective on $\bar{E} \backslash U(f)$, where $U(f)=\left\{\zeta \in \partial E: \lim _{z \rightarrow \zeta} f(z)=\infty\right\}$, and are such that $f^{\prime}(\zeta) \neq 0$ for $\zeta \in \partial E \backslash U(f)$.

To establish our main results we need the following Lemmas.

Lemma 2.2 (Miller and MocAnU [6]). Let $q$ be univalent in the unit disc $E$, and let $\theta$ and $\varphi$ be analytic in a domain $D$ containing $q(E)$, with 
$\varphi(w) \neq 0$ when $w \in q(E)$. Set $Q(z)=z q^{\prime}(z) \varphi(q(z)), h(z)=\theta(q(z)+Q(z)$ and suppose that:

(i) $Q$ is a starlike function in $E$,

(ii) $\mathfrak{R} e \frac{z h^{\prime}(z)}{Q(z)}>0, z \in E$.

If $p$ is analytic in $E$ with $p(0)=q(0), p(E) \subseteq D$ and

$$
\theta(p(z))+z p^{\prime}(z) \varphi(p(z)) \prec \theta(q(z))+z q^{\prime}(z) \varphi(q(z)),
$$

then $p(z) \prec q(z)$, and $q$ is the best dominant of (2.1).

Lemma 2.3 (Shanmugam ET AL. [13]). Let $\mu, \gamma \in \mathbb{C}$ with $\gamma \neq 0$, and let $q$ be a convex function in $E$ with

$$
\mathfrak{R} e\left(1+\frac{z q^{\prime \prime}(z)}{q^{\prime}(z)}\right)>\max \left\{0 ;-\mathfrak{R} e \frac{\mu}{\gamma}\right\}, z \in E .
$$

If $p$ is analytic in $E$ and

$$
\mu p(z)+\gamma z p^{\prime}(z) \prec \mu q(z)+\gamma z q^{\prime}(z),
$$

then $p(z) \prec q(z)$, and $q$ is the best dominant of (2.2).

Lemma 2.4 (BUlBOACA [5]). Let $q$ be a univalent function in the unit disc $E$, and let $\theta$ and $\varphi$ be analytic in a domain $D$ containing $q(E)$. Suppose that:

(i) $\mathfrak{R} e \frac{\theta^{\prime}(q(z))}{\varphi(q(z)}>0$, for $z \in E$,

(ii) $h(z)=z q^{\prime}(z) \varphi(q(z))$ is starlike in $E$.

If $p \in H[q(0), 1] \cap Q$ with $p(E) \subseteq D, \theta\left(p(z)+z p^{\prime}(z)\right) \varphi(p(z))$ is univalent in $E$, and

$$
\theta(q(z))+z q^{\prime}(z) \varphi(q(z)) \prec \theta(p(z))+z p^{\prime}(z) \varphi(p(z))
$$

then $q(z) \prec p(z)$, and $q$ is the best subordinant of (2.3).

Note that this result generalize a similar one obtained in [4].

Lemma 2.5 (Miller and Mocanu [7]). Let $q$ be convex in $E$ and let $\gamma \in \mathbb{C}$, with $\mathfrak{R} e \gamma>0$. If $p \in H[q(0), 1] \cap Q$ and $p(z)+\gamma z p^{\prime}(z)$ is univalent in $E$, then

$$
q(z)+\gamma z q^{\prime}(z) \prec p(z)+\gamma z p^{\prime}(z),
$$

implies $q(z) \prec p(z)$, and $q$ is the best subordinant of (2.4).

Lemma 2.6 (ROYSTER [10]). The function $q(z)=\frac{1}{(1-z)^{2 a b}}$ is univalent in $E$ if and only if $|2 a b-1| \leq 1$ or $|2 a b+1| \leq 1$. 


\section{Main results}

Theorem 3.1. Let $q$ be univalent in $E$, with $q(0)=1$, and suppose that

$$
\mathfrak{R} e\left(1+\frac{z q^{\prime \prime}(z)}{q(z)}\right)>\max \left\{0 ;-\mathfrak{R} e \frac{1}{\lambda}\right\}, \quad z \in E,
$$

where $\lambda \in \mathbb{C}^{*}=\mathbb{C} \backslash\{0\}$. If $f \in \mathcal{A}$ satisfies the subordination

$$
(1+\lambda)\left(\frac{2 z}{(f(z)-f(-z))}\right)-\lambda \frac{2 z^{2}\left(f^{\prime}(z)+f^{\prime}(-z)\right)}{((f(z)-f(-z)))^{2}} \prec q(z)+\lambda z q^{\prime}(z)
$$

then $\left(\frac{2 z}{(f(z)-f(-z))}\right) \prec q(z)$, and $q$ is the best dominant of Eq. (3.2).

Proof. Set

$$
\left(\frac{2 z}{(f(z)-f(-z))}\right)=h(z)
$$

where $h(z)$ is analytic in $E$ with $h(0)=1$.

A simple computation shows that

$$
(1+\lambda)\left(\frac{2 z}{(f(z)-f(-z))}\right)-\lambda \frac{2 z^{2}\left(f^{\prime}(z)+f^{\prime}(-z)\right)}{((f(z)-f(-z)))^{2}}=h(z)+\lambda z h^{\prime}(z),
$$

hence the subordination (3.2) is equivalent to $h(z)+\lambda z h^{\prime}(z) \prec q(z)+\lambda z q^{\prime}(z)$. Combining this last relation together with Lemma 2.2 for special case $\gamma=\lambda$ and $\mu=1$, we obtain our result.

Taking $q(z)=\frac{1+A z}{1+B z}$ in Theorem 3.1, where $-1 \leq B<A \leq 1$, the condition (3.1) reduces to

$$
\mathfrak{R} e \frac{1-B z}{1+B z}>\max \left\{0 ;-\mathfrak{R} e \frac{1}{\lambda}\right\}, \quad z \in E .
$$

It is easy to verify that the function $\varphi(\zeta)=\frac{(1-\zeta)}{(1+\zeta)}$, $|\zeta|<|B|$, is convex in $E$, and since $\varphi(\bar{\zeta})=\overline{\varphi(\zeta)}$ for all $|\zeta|<|B|$, it follows that $\varphi(E)$ is a convex domain symmetric with respect to the real axis, hence

$$
\inf \left\{\mathfrak{R} e \frac{1-B z}{1+B z}: z \in E\right\}=\frac{1-|B|}{1+|B|}>0 .
$$

Then, the inequality (3.4) is equivalent to $\mathfrak{R} e \frac{1}{\lambda} \geq \frac{|B|-1}{|B|+1}$, hence we have the following result. 
Corollary 3.2. Let $\lambda \in \mathbb{C}^{*},-1 \leq B<A \leq 1$ with $\max \left\{0 ;-\mathfrak{R} e \frac{1}{\lambda}\right\} \leq$ $\frac{1-|B|}{1+|B|}$. If $f \in \mathcal{A}$, and

$$
\begin{aligned}
& (1+\lambda)\left(\frac{2 z}{(f(z)-f(-z))}\right)-\lambda \frac{2 z^{2}\left(f^{\prime}(z)+f^{\prime}(-z)\right)}{((f(z)-f(-z)))^{2}} \\
& \prec \frac{1+A z}{1+B z}+\lambda \frac{(A-B) z}{(1+B z)^{2}},
\end{aligned}
$$

then

$$
\left(\frac{2 z}{(f(z)-f(-z))}\right) \prec \frac{1+A z}{1+B z}
$$

and $\frac{1+A z}{1+B z}$ is the best dominant of (3.6).

Example 3.3. Let $\lambda \in \mathbb{C}^{*}$ with $\mathfrak{R} e \frac{1}{\lambda} \geq 0$. If $f \in \mathcal{A}$, and

$$
(1+\lambda)\left(\frac{2 z}{(f(z)-f(-z))}\right)-\lambda \frac{2 z^{2}\left(f^{\prime}(z)+f^{\prime}(-z)\right)}{((f(z)-f(-z)))^{2}} \prec \frac{1+z}{1-z}+\lambda \frac{2 z}{(1-z)^{2}}
$$

then

$$
\left(\frac{2 z}{(f(z)-f(-z))}\right) \prec \frac{1+z}{1-z}
$$

and $\frac{1+z}{1-z}$ is the best dominant of (3.7).

Theorem 3.4. Let $q$ be univalent in $E$, with $q(0)=1$ and $q(z) \neq 0$ for all $z \in E$. Let $\gamma, \mu \in \mathbb{C}^{*}$ and $v, \eta \in \mathbb{C}$, with $v+\eta \neq 0$. Let $f \in \mathcal{A}$ and suppose that $f$ and $q$ satisfy the following conditions:

$$
\frac{2(v+\eta) z}{v z\left(f^{\prime}(z)+f^{\prime}(-z)\right)+\eta(f(z)-f(-z))} \neq 0, \quad z \in E,
$$

and

$$
\mathfrak{R} e\left(1+\frac{z q^{\prime \prime}(z)}{q^{\prime}(z)}-\frac{z q^{\prime}(z)}{q(z)}\right)>0, z \in E
$$

If

$$
\begin{aligned}
& 1+\gamma \mu\left[1-\frac{v z\left(z\left(f^{\prime}(z)+f^{\prime}(-z)\right)^{\prime}+\eta z\left(f^{\prime}(z)+f^{\prime}(-z)\right)\right.}{v z\left(f^{\prime}(z)+f^{\prime}(-z)\right)+\eta(f(z)-f(-z))}\right] \\
& \prec 1+\gamma \frac{z q^{\prime}(z)}{q(z)}
\end{aligned}
$$


then

$$
\left[\frac{2(v+\eta) z}{v z\left(f^{\prime}(z)+f^{\prime}(-z)\right)+\eta(f(z)-f(-z))}\right]^{\mu} \prec q(z),
$$

and $q$ is the best dominant of (3.10). The power is the principal one.

Proof. We begin by setting

$$
\left[\frac{2(v+\eta) z}{v z\left(f^{\prime}(z)+f^{\prime}(-z)\right)+\eta(f(z)-f(-z))}\right]^{\mu}=h(z), z \in E,
$$

where $h(z)$ is analytic in $E$ with $h(0)=1$. Differentiating Equation (3.11) logarithmically with respect to $z$, we have

$$
\mu\left[1-\frac{v z\left(z\left(f^{\prime}(z)+f^{\prime}(-z)\right)^{\prime}+\eta z\left(f^{\prime}(z)+f^{\prime}(-z)\right)\right.}{v z\left(f^{\prime}(z)+f^{\prime}(-z)\right)+\eta(f(z)-f(-z))}\right]=\frac{z h^{\prime}(z)}{h(z)} .
$$

To prove our result we use Lemma 2.1. Consider in this Lemma $\theta(w)=$ 1 and $\varphi(w)=\frac{\gamma}{w}$, then $\theta$ is analytic in $\mathbb{C}$ and $\varphi(w) \neq 0$ is analytic in $\mathbb{C}^{*}$. Also, if we let $Q(z)=z q^{\prime}(z) \varphi(q(z))=\gamma \frac{z q^{\prime}(z)}{q(z)}$, and $g(z)=\theta(q(z))+Q(z)=$ $1+\gamma \frac{z q^{\prime}(z)}{q(z)}$, then, since $Q(0)=0$ and $Q^{\prime}(0) \neq 0$, the assumption (3.9) would yield that $Q$ is a starlike function in $E$. From (3.9), we have

$$
\mathfrak{R} e \frac{z g^{\prime}(z)}{Q(z)}=\mathfrak{R} e\left(1+\frac{z q^{\prime \prime}(z)}{q^{\prime}(z)}-\frac{z q^{\prime}(z)}{q(z)}\right)>0, z \in E,
$$

and by using Lemma 2.1 we deduce that the subordination (3.10) implies that $h(z) \prec q(z)$, and the function $q$ is the best dominant of (3.10).

In particular, $v=0, \eta=\gamma=1$ and $q(z)=\frac{1+A z}{1+B z}$ in the above Theorem 3.4, it is easy to see that the assumption (3.9) holds whenever $-1 \leq$ $A<B \leq 1$, which leads to the next result:

Corollary 3.5. Let $-1 \leq A<B \leq 1$ and $\mu \in \mathbb{C}^{*}$. Let $f \in \mathcal{A}$ and suppose that $\frac{2 z}{(f(z)-f(-z))} \neq 0, z \in E$.

If

$$
1+\mu\left[1-\frac{z\left(f^{\prime}(z)+f^{\prime}(-z)\right)}{(f(z)-f(-z))}\right] \prec 1+\frac{(A-B) z}{(1+A z)(1+B z)},
$$

then

$$
\left(\frac{2 z}{(f(z)-f(-z))}\right)^{\mu} \prec \frac{1+A z}{1+B z}
$$

and $\frac{1+A z}{1+B z}$ is the best dominant of (3.12). The power is the principal one. 
Putting $v=0, \eta=1, \gamma=\frac{1}{a b}, a, b \in \mathbb{C}^{*}, \mu=a$, and $q(z)=\frac{1}{(1-z)^{2 a b}}$ in Theorem 3.4, then combining this together with Lemma 2.5, we have the next result.

Corollary 3.6. Let $a, b \in \mathbb{C}^{*}$ such that $|2 a b-1| \leq 1$ or $|2 a b+1| \leq 1$. Let $f \in \mathcal{A}$ and let $\frac{2 z}{(f(z)-f(-z))} \neq 0$, for all $z \in E$. If

$$
1+\frac{1}{b}\left(1-\frac{z\left(f^{\prime}(z)+f^{\prime}(-z)\right)}{(f(z)-f(-z))}\right) \prec \frac{1+z}{1-z}
$$

then

$$
\left(\frac{2 z}{(f(z)-f(-z))}\right)^{a} \prec \frac{1}{(1-z)^{2 a b}}
$$

and $\frac{1}{(1-z)^{2 a b}}$ is the best dominant of (3.13). The power is the principal one.

Putting $v=0, \eta=\gamma=1$ and $q(z)=(1+B z)^{\frac{\mu(A-B)}{B}},-1 \leq B<A \leq 1$, $B \neq 0$ in Theorem 3.4, and using Lemma 2.5, we have the next result.

Corollary 3.7. Let $-1 \leq B<A \leq 1$ with $B \neq 0$, and suppose that $\left|\frac{\mu(A-B)}{B-1}\right| \leq 1$ or $\left|\frac{\mu(A-B)}{B+1}\right| \leq 1$. Let $f \in \mathcal{A}$ such that $\frac{2 z}{(f(z)-f(-z))} \neq 0$, for all $z \in E$, and let $\mu \in \mathbb{C}^{*}$. If

$$
1+\mu\left(1-\frac{z\left(f^{\prime}(z)+f^{\prime}(-z)\right)}{(f(z)-f(-z))}\right) \prec \frac{1+[B+\mu(A-B)] z}{1+B z},
$$

then

$$
\left(\frac{2 z}{(f(z)-f(-z))}\right)^{\mu} \prec(1+B z)^{\frac{\mu(A-B)}{B}},
$$

and $(1+B z)^{\frac{\mu(A-B)}{B}}$ is the best dominant of (3.14). Here the power is the principal one.

By taking $v=0, \eta=1, \gamma=\frac{e^{i \lambda}}{a b \cos \lambda}, a, b \in \mathbb{C}^{*},|\lambda|<\frac{\pi}{2}, \mu=a$ and

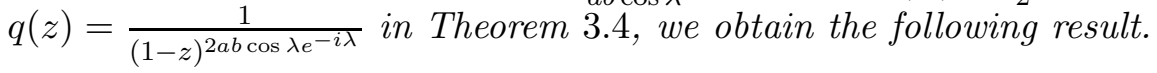

Corollary 3.8. Let $a, b \in \mathbb{C}^{*}$ and $|\lambda|<\frac{\pi}{2}$, and suppose that $\mid 2 a b \cos \lambda e^{-i \lambda}$ $1 \mid \leq 1$ or $\left|2 a b \cos \lambda e^{-i \lambda}+1\right| \leq 1$. Let $f \in \mathcal{A}$ such that $\frac{2 z}{(f(z)-f(-z))} \neq 0$, for all $z \in E$. If

$$
1+\frac{e^{i \lambda}}{b \cos \lambda}\left(1-\frac{z\left(f^{\prime}(z)+f^{\prime}(-z)\right)}{(f(z)-f(-z))}\right) \prec \frac{1+z}{1-z},
$$


then

$$
\left(\frac{2 z}{(f(z)-f(-z))}\right)^{a} \prec \frac{1}{(1-z)^{2 a b \cos \lambda e^{-i \lambda}}}
$$

and $\frac{1}{(1-z)^{2 a b \cos \lambda e^{-i \lambda}}}$ is the best dominant of (3.15). The power is the principal one.

Theorem 3.9. Let $q$ be univalent in $E$ with $q(0)=1$, let $\mu, \gamma \in \mathbb{C}^{*}$, and let $\mu, \gamma \in \mathbb{C}^{*}$ and let $\delta, \Omega, v, \eta \in \mathbb{C}$ with $v+\eta \neq 0$. Let $f \in \mathcal{A}$ and suppose that $f$ and $q$ satisfy the next two conditions:

$$
\begin{aligned}
& \frac{2(v+\eta) z}{v z\left(f^{\prime}(z)+f^{\prime}(-z)\right)+\eta(f(z)-f(-z))} \neq 0, z \in E, \\
& \mathfrak{R} e\left(1+\frac{z q^{\prime \prime}(z)}{q^{\prime}(z)}\right)>\max \left\{0 ;-\mathfrak{R} e \frac{\delta}{\gamma}\right\}, z \in E .
\end{aligned}
$$

If

$$
\begin{aligned}
\psi(z) & \equiv\left[\frac{2(v+\eta) z}{v z\left(f^{\prime}(z)+f^{\prime}(-z)\right)+\eta(f(z)-f(-z))}\right]^{\mu} \\
& {\left[\delta+\gamma \mu\left(1-\frac{v z\left(z\left(f^{\prime}(z)+f^{\prime}(-z)\right)^{\prime}+\eta z\left(f^{\prime}(z)+f^{\prime}(-z)\right)\right.}{v z\left(f^{\prime}(z)+f^{\prime}(-z)\right)+\eta(f(z)-f(-z))}\right)\right]+\Omega, }
\end{aligned}
$$

and

$$
\psi(z) \prec \delta q(z)+\gamma z q^{\prime}(z)+\Omega
$$

then

$$
\left[\frac{2(v+\eta) z}{v z\left(f^{\prime}(z)+f^{\prime}(-z)\right)+\eta(f(z)-f(-z))}\right]^{\mu} \prec q(z),
$$

and $q$ is the best dominant of (3.19). All the powers are the principal ones.

Proof. We begin by setting

$$
\left[\frac{2(v+\eta) z}{v z\left(f^{\prime}(z)+f^{\prime}(-z)\right)+\eta(f(z)-f(-z))}\right]^{\mu}=h(z) .
$$

Then $h(z)$ is analytic in $E$ with $h(0)=1$. Logarithmic differentiating of (3.20) yields

$$
\mu\left(1-\frac{v z\left(z\left(f^{\prime}(z)+f^{\prime}(-z)\right)^{\prime}+\eta z\left(f^{\prime}(z)+f^{\prime}(-z)\right)\right.}{v z\left(f^{\prime}(z)+f^{\prime}(-z)\right)+\eta(f(z)-f(-z))}\right)=\frac{z h^{\prime}(z)}{h(z)},
$$


and hence

$$
\mu h(z)\left(1-\frac{v z\left(z\left(f^{\prime}(z)+f^{\prime}(-z)\right)^{\prime}+\eta z\left(f^{\prime}(z)+f^{\prime}(-z)\right)\right.}{v z\left(f^{\prime}(z)+f^{\prime}(-z)\right)+\eta(f(z)-f(-z))}\right)=z h^{\prime}(z) .
$$

Let us consider the functions:

$$
\begin{aligned}
& \theta(w)=\delta w+\Omega, \quad \varphi(w)=\gamma, w \in \mathbb{C}, \\
& Q(z)=z q^{\prime}(z) \varphi\left(q(z)=\gamma z q^{\prime}(z), z \in E,\right.
\end{aligned}
$$

and $g(z)=\theta\left(q(z)+Q(z)=\delta q(z)+\gamma z q^{\prime}(z)+\Omega, z \in E\right.$. From the assumption (3.17) we see that $Q$ is starlike in $E$ and, that

$$
\mathfrak{R} e \frac{z g^{\prime}(z)}{Q(z)}=\mathfrak{R} e\left(\frac{\delta}{\gamma}+1+\frac{z q^{\prime \prime}(z)}{q^{\prime}(z)}\right)>0, z \in E,
$$

thus, by applying Lemma 2.1 this completes the proof.

Taking $q(z)=\frac{(1+A z)}{(1+B z)}$ in Theorem 3.7, where $-1 \leq B<A \leq 1$ and according to (3.5), the condition (3.17) becomes

$$
\max \left\{0 ;-\mathfrak{R} e \frac{\delta}{\gamma}\right\} \leq \frac{1-|B|}{1+|B|}
$$

Hence, for the particular case $v=1=\gamma, \eta=0$, we have the following result:

Corollary 3.10. Let $-1 \leq B<A \leq 1$ and let $\delta \in \mathbb{C}$ with

$$
\max \{0 ;-\mathfrak{R} e \delta\} \leq \frac{1-|B|}{1+|B|} .
$$

Let $f \in \mathcal{A}$ and suppose that $\frac{2}{\left(f^{\prime}(z)+f^{\prime}(-z)\right)} \neq 0, z \in E$ and let $\mu \in \mathbb{C}^{*}$. If

$$
\begin{aligned}
& {\left[\frac{2}{\left(f^{\prime}(z)+f^{\prime}(-z)\right)}\right]^{\mu}\left[\delta+\mu\left(1-\frac{z\left(f^{\prime}(z)+f^{\prime}(-z)\right)}{f(z)-f(-z)}\right)\right]+\Omega} \\
& \prec \delta \frac{1+A z}{1+B z}+\Omega+\frac{z(A-B)}{(1+B z)^{2}}
\end{aligned}
$$

then

$$
\left(\frac{2}{\left(f^{\prime}(z)+f^{\prime}(-z)\right)}\right)^{\mu} \prec \frac{1+A z}{1+B z}
$$

and $\frac{1+A z}{1+B z}$ is the best dominant of (3.21). All the powers are the principal ones. 
Taking $\gamma=\eta=1, v=0$ and $q(z)=\frac{1+z}{1-z}$ in Theorem 3.7, we obtain the next result.

Corollary 3.11. Let $f \in \mathcal{A}$ such that $\frac{2 z}{f(z)-f(-z)} \neq 0$ for all $z \in E$, and let $\mu \in \mathbb{C}^{*}$. If

$$
\begin{aligned}
& {\left[\frac{2 z}{f(z)-f(-z)}\right]^{\mu}\left[\delta+\mu\left(1-\frac{z\left(f^{\prime}(z)+f^{\prime}(-z)\right)}{f(z)-f(-z)}\right)\right]+\Omega} \\
& \prec \delta \frac{1+z}{1-z}+\Omega+\frac{2 z}{(1-z)^{2}}
\end{aligned}
$$

then $\left[\frac{2 z}{f(z)-f(-z)}\right]^{\mu} \prec \frac{1+z}{1-z}$, and $\frac{1+z}{1-z}$ is the best dominant of (3.22). All the powers are the principal ones.

\section{Superordination and sandwich theorems}

Theorem 4.1. Let $q$ be convex in $E$ with $q(0)=1$, let $\lambda \in \mathbb{C}^{*}$ with $\Re e \lambda>0$. Let $f \in \mathcal{A}$ and suppose that $\frac{2 z}{f(z)-f(-z)} \in H[q(0), 1] \cap Q$. If the function

$$
(1+\lambda)\left(\frac{2 z}{(f(z)-f(-z))}\right)-\lambda \frac{2 z^{2}\left(f^{\prime}(z)+f^{\prime}(-z)\right)}{((f(z)-f(-z)))^{2}}
$$

is univalent in the unit disc $E$, and

$$
q(z)+\lambda z q^{\prime}(z) \prec(1+\lambda)\left(\frac{2 z}{(f(z)-f(-z))}\right)-\lambda \frac{2 z^{2}\left(f^{\prime}(z)+f^{\prime}(-z)\right)}{((f(z)-f(-z)))^{2}}
$$

then

$$
q(z) \prec\left(\frac{2 z}{(f(z)-f(-z))}\right),
$$

and $q$ is the best subordinant of (4.1).

Proof. Set

$$
\left(\frac{2 z}{(f(z)-f(-z))}\right)=h(z), z \in E .
$$

Then $h(z)$ is analytic in $E$ with $h(0)=1$. Taking logarithmic differentiation with respect $z$, we have

$$
1-z\left(\frac{f^{\prime}(z)+f(-z)}{f(z)-f(-z)}\right)=\frac{z h^{\prime}(z)}{h(z)} .
$$


A simple computations show that

$$
h(z)+\lambda z h^{\prime}(z)=(1+\lambda)\left(\frac{2 z}{(f(z)-f(-z))}\right)-\lambda \frac{2 z^{2}\left(f^{\prime}(z)+f^{\prime}(-z)\right)}{((f(z)-f(-z)))^{2}},
$$

and now, by using Lemma 2.4, this completes the proof.

Taking $q(z)=\frac{1+A z}{1+B z}$ in Theorem 4.1 , where $-1 \leq B<A \leq 1$, we obtain the next result.

Corollary 4.2. Let $q$ be convex in $E$ with $q(0)=1$, let $\lambda \in \mathbb{C}^{*}$ with $\Re e \lambda>0$. Let $f \in \mathcal{A}$ suppose that $\frac{2 z}{f(z)-f(-z)} \in H[q(0), 1] \cap Q$. If the function

$$
(1+\lambda)\left(\frac{2 z}{(f(z)-f(-z))}\right)-\lambda \frac{2 z^{2}\left(f^{\prime}(z)+f^{\prime}(-z)\right)}{((f(z)-f(-z)))^{2}}
$$

is univalent in the unit disc $E$, and

$$
\begin{aligned}
& \frac{1+A z}{1+B z}+\frac{\lambda(A-B) z}{(1+B z)^{2}} \prec(1+\lambda)\left(\frac{2 z}{(f(z)-f(-z))}\right) \\
& -\lambda \frac{2 z^{2}\left(f^{\prime}(z)+f^{\prime}(-z)\right)}{((f(z)-f(-z)))^{2}},
\end{aligned}
$$

then

$$
\frac{1+A z}{1+B z} \prec\left(\frac{2 z}{(f(z)-f(-z))}\right),
$$

and $\frac{1+A z}{1+B z}$ is the best subordinant of (4.3), where $-1 \leq B<A \leq 1$.

Using the same techniques as in Theorem 3.8, and then applying Lemma 2.3, we have the following theorem.

Theorem 4.3. Let $q$ be convex in $E$ with $q(0)=1$, let $\mu, \gamma \in \mathbb{C}^{*}$, and let $\delta, \Omega, v, \eta \in \mathbb{C}$ with $v+\eta \neq 0$ and $\mathfrak{R} e \frac{\delta}{\gamma}>0$. Let $f \in \mathcal{A}$ and suppose that $f$ satisfies the following conditions:

$$
\frac{2(v+\eta) z}{v z\left(f^{\prime}(z)+f^{\prime}(-z)\right)+\eta(f(z)-f(-z))} \neq 0, z \in E,
$$

and

$$
\left[\frac{2(v+\eta) z}{v z\left(f^{\prime}(z)+f^{\prime}(-z)\right)+\eta(f(z)-f(-z))}\right]^{\mu} \in H[q(0), 1] \cap Q .
$$


If the function $\psi$ given by equation (3.18) is univalent in $E$, and

$$
\delta q(z)+\gamma z q^{\prime}(z)+\Omega \prec \psi(z),
$$

then

$$
q(z) \prec\left[\frac{2(v+\eta) z}{v z\left(f^{\prime}(z)+f^{\prime}(-z)\right)+\eta(f(z)-f(-z))}\right]^{\mu},
$$

and $q$ is the best subordinate of (4.4) (all powers are the principal ones).

Note that by combining Theorem 3.1 with Theorem 4.1 and Theorem 3.10 with Theorem 4.3, we have, respectively, the following two sandwich theorems:

Theorem 4.4. Let $q_{1}$ and $q_{2}$ be two convex functions in $E$ with $q_{1}(0)=$ $q_{2}(0)=1$, let $\lambda \in \mathbb{C}^{*}$ with $\Re e \lambda>0$. Let $f \in \mathcal{A}$ and suppose that $\frac{2 z}{f(z)-f(-z)} \in$ $H[q(0), 1] \cap Q$. If the function

$$
(1+\lambda)\left(\frac{2 z}{(f(z)-f(-z))}\right)-\lambda \frac{2 z^{2}\left(f^{\prime}(z)+f^{\prime}(-z)\right)}{((f(z)-f(-z)))^{2}}
$$

is univalent in the unit disc $E$, and

$$
\begin{aligned}
q_{1}(z)+\lambda z q_{1}^{\prime}(z) & \prec(1+\lambda)\left(\frac{2 z}{(f(z)-f(-z))}\right)-\lambda \frac{2 z^{2}\left(f^{\prime}(z)+f^{\prime}(-z)\right)}{((f(z)-f(-z)))^{2}} \\
& \prec q_{2}(z)+\lambda z q_{2}^{\prime}(z),
\end{aligned}
$$

then

$$
q_{1}(z) \prec\left(\frac{2 z}{(f(z)-f(-z))}\right) \prec q_{2}(z),
$$

and $q_{1}$ and $q_{2}$ are, respectively, the best subordinate and the best dominant of (4.5).

Theorem 4.5. Let $q_{1}$ and $q_{2}$ be two convex functions in $E$ with $q_{1}(0)=$ $q_{2}(0)=1$, let $\mu, \gamma \in \mathbb{C}^{*}$, and let $\delta, \Omega, v, \eta \in \mathbb{C}$ with $v+\eta \neq 0$ and $\mathfrak{R} e \frac{\delta}{\gamma}>0$. Let $f \in \mathcal{A}$ satisfy the following conditions:

$$
\left[\frac{2(v+\eta) z}{v z\left(f^{\prime}(z)+f^{\prime}(-z)\right)+\eta(f(z)-f(-z))}\right] \neq 0, z \in E,
$$

and

$$
\left[\frac{2(v+\eta) z}{v z\left(f^{\prime}(z)+f^{\prime}(-z)\right)+\eta(f(z)-f(-z))}\right]^{\mu} \in H[q(0), 1] \cap Q .
$$


If the function $\psi$ given by (3.18) is univalent in $E$, and

$$
\delta q_{1}(z)+\gamma z q_{1}^{\prime}(z)+\Omega \prec \psi(z) \prec \delta q_{2}(z)+\gamma z q_{2}^{\prime}(z)+\Omega,
$$

then

$$
q_{1}(z) \prec\left[\frac{2(v+\eta) z}{v z\left(f^{\prime}(z)+f^{\prime}(-z)\right)+\eta(f(z)-f(-z))}\right]^{\mu} \prec q_{2}(z),
$$

and $q_{1}$ and $q_{2}$ are, respectively, the best subordinate and the best dominant of (4.6) (all the powers are the principal ones).

\section{REFERENCES}


tivalent functions defined by certain integral operator, J. Franklin Inst., 347 (2010), 641-653.

2. Aouf, M.K.; Al-Oboudi, F.M.; Haidan, M.M. - On some results for $\lambda$-spirallike and $\lambda$-Robertson functions of complex order, Publ. Inst. Math. (Beograd) (N.S.), 77 (2005), 93-98.

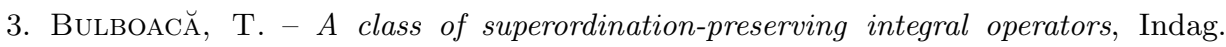
Math. (N.S.), 13 (2002), 301-311.

4. Bulboac $\breve{,}$, T. - Classes of first-order differential superordinations, Demonstratio Math., 35 (2002), 287-292.

5. Bulboaç̆, T. - Differential Subordination and Superordinations, Recent Results, House of Scientific Book Publ, Cluj-Napoca, 2005.

6. Miller, S.S.; Mocanu, P.T. - Differential Subordinations. Theory and Applications, Monographs and Textbooks in Pure and Applied Mathematics, 225, Marcel Dekker, Inc., New York, 2000.

7. Miller, S.S.; Mocanu, P.T. - Subordinants of differential superordinations, Complex Var. Theory Appl., 48 (2003), 815-826.

8. Obradović, M.; Aouf, M.K.; Owa, S. - On some results for starlike functions of complex order, Publ. Inst. Math. (Beograd) (N.S.), 46 (1989), 79-85.

9. Obradović, M.; Owa, S. - On certain properties for some classes of starlike functions, J. Math. Anal. Appl., 145 (1990), 357-364.

10. Royster, W.C. - On the univalence of a certain integral, Michigan Math. J., 12 (1965), 385-387. 
11. Shams, S.; Kulkarni, S.R.; Jahangiri, J.M. - Subordination properties of p-valent functions defined by integral operators, Int. J. Math. Math. Sci. 2006, Art. ID 94572, $3 \mathrm{pp}$.

12. Shammugam, T.N.; Ramachandran, C.; Darus, M.; Sivasubramanian, S. Differential sandwich theorems for some subclasses of analytic functions involving a linear operator, Acta Math. Univ. Comenian. (N.S.), 76 (2007), 287-294.

13. Shanmugam, T.N.; Sivasubramanian, S.; Silverman, H. - On sandwich theorems for some classes of analytic functions, Int. J. Math. Math. Sci., 2006, Art. ID 29684, $13 \mathrm{pp}$.

14. SAKaguchi, K. - On a certain univalent mapping, J. Math. Soc. Japan, 11 (1959), 72-75.

Received: 3.I.2012

Revised: 8.IV.2012

Accepted: 18.IV.2012
Department of Basic Sciences, University of Engineering and Technology, Peshawar, PAKISTAN ali7887@gmail.com 